\title{
基于深度卷积网络与尺度不变特征变换的行人跟踪 框架
}

陈凯，宋晓*，刘敬

北京航空航天大学自动化科学与电气工程学院, 北京 100191

*通信作者. E-mail: songxiao@buaa.edu.cn

收稿日期: 2017-12-27; 接受日期: 2018-03-18; 网络出版日期: 2018-07-17

国家自然科学基金 (批准号: 61473013) 资助项目

摘要 在计算机视觉领域已经提出了很多物体跟踪算法和多人跟踪算法, 但是遮挡一直是跟踪领 域一个具有较大挑战性的问题. 为了实现在多种遮挡情况下的行人跟踪, 本文提出了一种基于深度 学习的行人跟踪框架: 根据物体检测算法 Faster R-CNN 训练了一个行人检测算法作为行人跟踪的 搜索机制, 相比于梯度下降算法这个机制在缩小搜索范围的同时提高了准确度; 利用颜色直方图和 尺度不变特征相结合作为目标的表示方式, 在目标匹配的过程中, 基于 FCN 图像语义分割算法训 练了一个针对行人全卷积网络来提取目标模型中的行人信息以便于去除背景的噪声影响. 在数据库 OTB 上与其他跟踪方法进行了比较, 结果显示所提出方法比其他跟踪器在有遮挡的情况下提高了 跟踪的准确率和成功率.

关键词 Faster R-CNN, SIFT, FCN, 图像语义分割算法, 颜色直方图

\section{1 引言}

行人跟踪是计算机视觉领域的一个重要问题, 在很多应用领域都有广泛的应用, 比如无人车、机 器人、视频监控等. 我们最终取得的效果是帮助无人车、机器人、警察在具有遮挡的复杂监控画面中 跟踪到目标. 然而传统的目标跟踪算法跟踪的场景过于简单, 行人跟踪领域还存在很多挑战, 例如光照 强度、行人姿态、变化、遮挡等 ${ }^{[1 \sim 5]}$. 尤其是物体对行人的遮挡以及行人之间的相互遮挡是一直以来 一个具有很大挑战性的难题. 目前在目标跟踪领域已经提出了很多优秀的算法, 这些算法可以被分为 产生式和判别式两大类. 尽管这两大类跟踪算法在目标跟踪方面都取得了很好的效果, 但是一旦有较 大的部分遮挡或者长时间遮挡的存在, 它们就会产生漂移, 甚至丢失跟踪目标.

现有的优秀产生式和判别式算法由于采用均值漂移 [6,7] 和粒子滤波 ${ }^{[8]}$ 等算法作为搜索机制, 这 些搜索机制都是由上一帧目标的位置作为初始位置迭代搜索目标, 当有遮挡存在的情况下, 目标模型

\footnotetext{
引用格式: 陈凯, 宋晓, 刘敬. 基于深度卷积网络与尺度不变特征变换的行人跟踪框架. 中国科学: 信息科学, 2018, 48: 841-855, doi: 10.1360/N112017-00286

Chen K, Song X, Liu J. Pedestrian tracking framework based on deep convolution network and SIFT (in Chinese). Sci Sin Inform, 2018, 48: 841-855, doi: 10.1360/N112017-00286
} 
因为参杂遮挡物信息, 导致目标模型逐渐发生改变, 以至于跟踪发生漂移. 为了解决行人跟踪过程中 各种遮挡问题, 本文所提出的行人跟踪框架, 预先训练一个行人检测器作为目标搜索机制, 这样就使得 目标模型更多包含行人信息, 然后用图像特征算法来区分我们的目标和其他行人的跟踪思路.

本文在以下 3 方面为行人跟踪做出了贡献:

(1) 为了能够在有遮挡的情况下快速准确地跟踪到目标行人, 使用能够达到近乎实时的高精度检 测的 Faster R-CNN ${ }^{[9]}$ 作为搜索机制, 采用支持简单快速对比的 RGB 颜色直方图和具有对旋转、尺 度缩放、亮度变化保持不变性, 对视角变化、仿射变换、噪声也保持一定程度的稳定性的 SIFT ${ }^{[10]}$ 作 为目标表示方式.

(2) 基于颜色直方图 ${ }^{[11]}$, 本文提出了一种新的相似度计算方法提高了目标之间的区分度, 增加了 颜色直方图匹配的准确度.

(3) 在有遮挡的情况下, 为了更好地利用 SIFT 特征匹配来定位跟踪目标, 基于 VGG16 ${ }^{[12]}$ 深度卷 积网络改造了一个全卷积网络 $(\mathrm{FCN})^{[11]}$ 用于区分背景和行人, 以便在进行 SIFT 特征匹配的时候去 除背景的影响.

本文的其他部分主要由以下组成. 在第 2 节介绍一些相关的工作. 在第 3 节详细介绍本文提出的 跟踪框架, 并且在第 4 节用真实的数据评估本文的方法和其他跟踪算法. 在第 5 节对论文进行总结.

\section{2 相关工作}

计算机视觉在目标检测、图像特征表示、目标跟踪等方面已经获得广泛的发展. 在目标跟踪领域 已经产生了很多优秀的跟踪算法, 例如 $\mathrm{CT}^{[13]}, \mathrm{STC}^{[14]}, \mathrm{CSK}^{[15]}, \mathrm{KCF}^{[16]}, \mathrm{OCT}^{[\mathrm{KCF}}{ }^{[17]}$ 等, 这些算 法在目标跟踪领域都有突破性的创新.

CT 跟踪是矩阵稀疏表示的实际应用, 依旧采用不同范围的正负样本采集, 然后用朴素 Bayes 分 类器分类, 巧妙之处在于, 对于 ROI 区域图像的稀疏表示, 作者找到了一个 $N(0,1)$ 分布的非常稀疏 的随机测量矩阵, 将其与 ROI 图像卷积就是对 ROI 图像的稀疏表示, 这种卷积等价于矩形滤波, 这与 HAAR 特征一样, 于是, 作者巧妙地将 ROI 的稀疏表示用 HAAR 特征完成降维. 同时, 多尺度表示也 是不同尺度的 HAAR 矩形. HAAR 特征, 随机取一定数量, 满足多尺度. 样本选择上, 以目标为参考, 周围样本与目标中心相距 $0 \sim r$ 为正样本, $a \sim b$ 为负样本, 其中 $r<a<b$. 这种样本模型漂移, 这也 是 $\mathrm{CT}$ 容易漂移的原因.

STC 是一个简单快速而且鲁棒的算法, 它利用稠密的空时场景模型来进行跟踪. 在 Bayes 框架下, 它利用目标和目标局部的稠密信息的空时关系来建模. 置信图在被计算时考虑了上一帧目标的位置的 先验信息, 这有效地减轻了目标位置的模糊. STC 跟踪器使用了最简单的灰度特征, 但是灰度并不能 很好地对外观进行描述. 这里可以改进为其他比较好的特征 (Color name 或者 Hog), 但是就会遇到 多通道特征融合的问题. 一般的 Tracking-by-Detection 跟踪算法基本都不能实现尺度的变化, 而 STC 跟踪器就提出了一种有效的尺度变化方案, 通过连续两帧的目标最佳位置处的置信值的比值来计算当 前帧中目标的估计尺度. 为了不引入噪声和避免过度敏感的自适应引入连续 $n$ 帧的平均估计尺度, 最 后通过滤波获得最终的目标估计尺度. STC 跟踪器对光照变化、尺度变化 (实际测试下来没那么好)、 姿势变化、遮挡、旋转、背景杂乱和突然运动的视频都有较好的跟踪, 但对刚性形变、出视角和低分 辨率的视频效果不佳.

KCF 是对 CSK 跟踪器的优化, 这里简单介绍一下 CSK 跟踪器的主要思想. CSK 跟踪器最大亮 点就是提出了利用循环移位的方法进行稠密采样并结合 FFT 快速地进行分类器的训练. 稠密采样的 
采样方式能提取目标的所有信息, 这对目标的跟踪至关重要. 虽然 CSK 的速度很快, 但是 CSK 只是 简单地使用了灰度特征, 对目标的外观描述能力显然不足. 对此作者改进了 CSK 提出了 $\mathrm{KCF}$, 从原来 的单通道灰度特征换成了多通道 $\operatorname{Hog}$ 特征. KCF 算法通过核函数对多通道的 Hog 特征进行了融合, 使得训练所得的分类器对待检测目标的解释力更强. 核心相关滤波器 (KCF) 是最先进的对象跟踪器 之一. 然而, 它没有合理地模拟跟踪过程中相关响应的分布, 这可能导致漂移问题, 特别是当目标由于 闭塞, 相机抖动和/或变形而出现显著的外观变化时.

输出约束传递 (OCT) 方法, 通过建模 Bayes 优化框架中的相关响应分布能够减轻漂移问题. OCT 基于对目标图像的相关响应遵循 Gauss 分布的合理假设, 利用它们选择训练样本并减少模型不确定 性. OCT 植根于一种将数据分布转移到优化变量约束的新理论, 从而形成一个有效的框架来计算相关 性滤波. 通常使用的跟踪基准测试表明, OCT 显著提高了 $\mathrm{KCF}$, 并且比其他最先进的跟踪器具有更好 的性能. 但是 OCT-KCF 在遇到遮挡, 特别是全部遮挡时存在严重的漂移, 并且在处理像素高的图像 时速度明显很慢, 实时性比较差.

经过实际的实验表明, 这些跟踪器对遮挡非常敏感. 在本文的行人遮挡实验中, 这些跟踪器在行 人被障碍物部分遮挡、长时间遮挡、行人相互遮挡的情况下, 存在严重的跟踪漂移问题. 为了解决这些 遮挡问题, 本文在目标搜索机制、目标表示和目标匹配方面进行了创新的研究.

针对行人跟踪, 本文采用行人检测模型作为目标搜索机制. 在文献 [9] 中, 作者在 Fast R-CNN ${ }^{[18]}$ 的基础上提出了 Faster R-CNN, 它主要解决两个问题: 第一, 提出区域建议网络 RPN ${ }^{[19]}$, 快速生成候 选区域; 第二, 通过交替训练, 使 RPN 和 Fast R-CNN 网络共享参数. Faster R-CNN 创造性地采用卷 积网络自行产生建议框, 并且和目标检测网络共享卷积网络, 使得建议框数目从原有的约 2000 个减少 为 300 个, 这使得目标检测几乎达到实时, 并且建议框的质量也有本质的提高. 基于这两点, 本文参考 Faster R-CNN 的思路训练了一个新的行人检测模型, 并且在行人遮挡检测方面针对训练数据进行了 优化.

目标表示方面, 本文采用了颜色直方图和 SIFT 特征相结合的算法. 颜色直方图中的数值描述了 该图像中关于颜色的数量特征, 可以反映图像颜色的统计分布和基本色调. 直方图只包含了该图像中 某一颜色值出现的频数, 任一幅图像都能唯一的给出一幅与它对应的直方图, 但不同的图像可能有相 同的颜色分布, 从而就具有相同的直方图, 因此直方图与图像是一对多的关系. 因此比较简单的场景中 可以用颜色直方图对比来帮助我们追踪目标, 但是一旦出现遮挡或光照、形状等因素的改变, 颜色直 方图就不适用了, 这时要利用 SIFT 特征匹配. Lowe ${ }^{[10]}$ 提出了尺度不变特征转换, 局部影像特征的描 述与侦测可以帮助辨识物体, SIFT 特征是基于物体上的一些局部外观的兴趣点, 与影像的大小和旋转 无关, 对于光线、噪声、视角改变的容忍度也相当高. 基于这些特性, 它们是高度显著而且相对容易截 取, 在数目庞大的特征数据库中, 很容易辨识物体而且鲜有误认. 使用 SIFT 特征描述对于部分物体遮 蔽的帧率也相当高, 甚至只需要 3 个以上的 SIFT 物体特征就足以计算出位置与方位. 在现今的电脑 硬件速度下和小型的特征数据库条件下, 辨识速度可接近即时运算. SIFT 特征的信息量大, 适合在海 量数据库中快速准确匹配.

在进行行人 SIFT 特征匹配的时候, 目标模型的背景中往往含有很多, 为了消除这些噪声点的影 响, 本文采用 FCN 图像语义分割算法消除噪声. 文献 [11] 中将 FCN 应用到了图像语义分割领域. 图 像语义分割, 就是对一张图片上的所有像素点进行分类. 以往的 CNN 都是对整张图片进行分类, 不能 识别图片中特定部分的物体, 而全卷积网络是对一张图片中的每个像素进行分类, 以此达到对图片特 定部分进行分类的效果. 本文根据文献 [11] 的思路基于 VGG16 卷积模型构建了一个全卷积网络. 


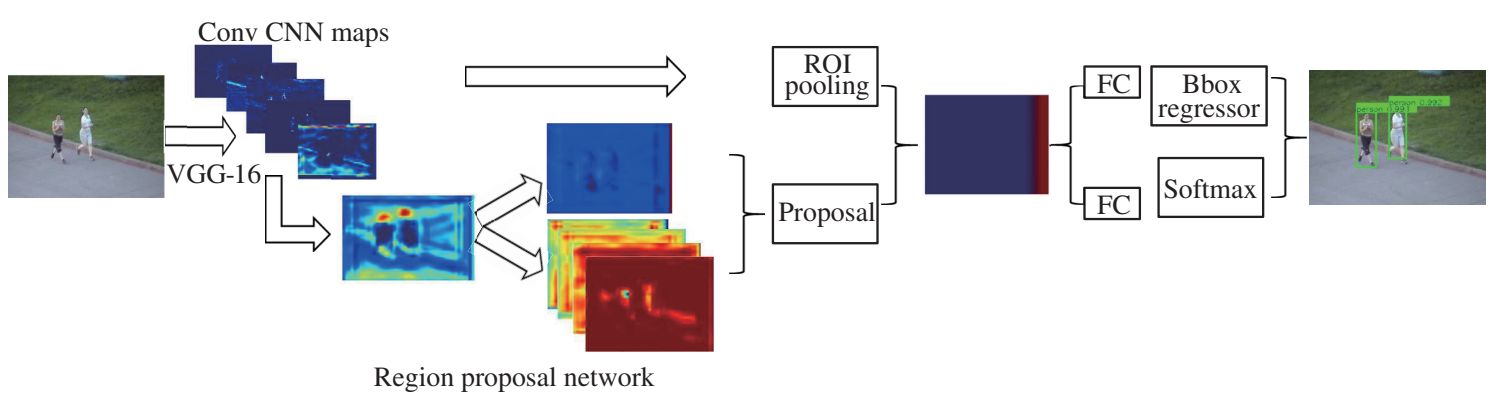

图 1 (网络版彩图) 基于 Faster R-CNN 的行人检测系统框架

Figure 1 (Color online) The architecture of the Faster R-CNN based pedestrian detection system

\section{3 行人跟踪框架介绍}

本节从 3 个主要的模块介绍本文的行人跟踪框架: 行人搜索机制、目标表示方式和目标匹配方式.

\section{1 目标搜索机制}

我们需要准确迅速地定位行人的候选位置, 行人检测算法 Faster R-CNN 可以迅速准确地检测出 视频中每一帧图像中的所有行人, 并且它可以解决传统跟踪算法每次跟踪误差的累计造成目标跟踪的 漂移.

但是 Faster R-CNN 检测部分遮挡的行人准确率不高, 而且在文献 [9] 中对其实现方法讲解得不 多. 因此, 针对存在遮挡的行人检测, 我们基于数据集 VOC2012 扩充了更多地存在 $50 \%$ 以下遮挡的 行人数据, 提高了 Faster R-CNN 对部分遮挡的行人的检测成功率.

基于 Faster R-CNN, 本文提出的行人检测模型有 3 阶段架构. 行人 Faster R-CNN 架构如图 1 所示.

在第 1 阶段, 区域提议网络 (RPN) 用于为每个图像生成提出的区域. 在本文中, 采用 RPN 方法 获取区域提案. 不同于其他类别无关的方法, 例如选择性搜索或边框, RPN 使用预训练的卷积神经网 络 $(\mathrm{CNN})$ 来分割图像. 它需要任意大小的图像作为输入, 并输出多个矩形区域提案. 由于 CNN 的灵 活性高, 因此无需使用类似的行为类对象来生成专门的区域提案.

第 2 阶段使用基于 Fast R-CNN 的深度卷积网络来提取许多地区提案的特征. 具有 13 个卷积层 的 Simonyan 和 Zisserman 模型 VGG-16 在 RPN 中用作特征提取器. 然后, 使用由最后一层生成的特 征图上的 $3 \times 3$ 窗口的滑动窗网络来获得具有 512 个维度的固定尺寸向量. 在每个窗口位置, RPN 生 成 9 个区域提案 (称为针点), 具有 3 个不同的比例和宽高比, 以提供强大的平移不变属性. 生成区域 提案的过程可以被认为是空间金字塔池化网络 (SPPNet) ${ }^{[20]}$ 的一种特殊情况. 然后将所得到的矢量 输入到两个卷积层, 框坐标回归层和分类层. 框坐标回归层用于预测每个坐标的偏移. 它为每个边界 框输出所有 9 个针点的左上角和右下角坐标. 因此, 它具有 $4 \times 9$ 维输出层. 对于分类层, 输出是每个 针的行人和背景得分. 因此, 该层有 $2 \times 9$ 个输出.

最后一个阶段训练一个 2 级 softmax 分类器来检测行人. Fast R-CNN 用于检测每个图像中的行 人. 它将图像和相应的区域提案作为输入, 并使用两个兄弟 softmax 分类器来生成边界框和类分数. 该图像由 VGG-16 网络首先处理, 以生成特征图. 这些映射是最终层的输出, 并被馈送到利用区域提 案的兴趣区域 (RoI) 池层中. 该池层首先将特征图映射到每个区域提案中, 使得特征位于特征图的相 同位置. 然后, 它使用最大池化将特征图的这一部分转换成大小为 $7 \times 7$ 的小区域. RoI 是一个四元组 

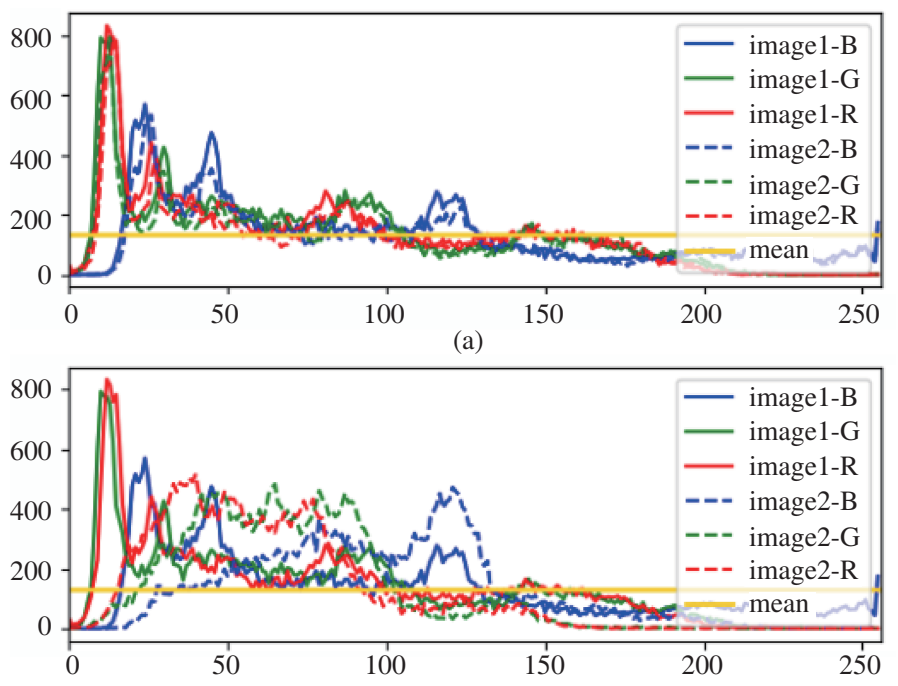

(b)

图 2 (网络版彩图) 两张图像的颜色直方图对比

Figure 2 (Color online) Comparison of color histograms of two images. Different pictures of (a) the same and (b) the different pedestrian

$(x, y, w, h)$ 的矩形窗口, 表示左上角坐标、宽度和高度. 每个 $h \times w$ 区域提案使用大小约为 $h / 7 \times w / 7$ 的子窗口进行最大合并. RoI 层也是一个只有一个级别的 SPPNet 的特殊情况. RoI 被映射到使用两 个网络的固定大小的向量, 并且这被输入到两个 softmax 分类器以获得最终确认分数和修正的边界框 坐标.

\section{2 目标表示}

在任何视觉跟踪器中, 目标表示都是一个主要的组成部分, 并且大量的方法已经被提出 ${ }^{[21]}$. 由于 Lucas 和 Kanade ${ }^{[22,23]}$ 的开创性研究, 全局模板 (原始灰度值) 在跟踪中已经被广泛地使用 ${ }^{[24 ~ 26]}$. 除 了模板, 许多其他视觉特征也已经被用于跟踪算法, 比如颜色直方图、尺度不变特征 (SIFT)、方向梯

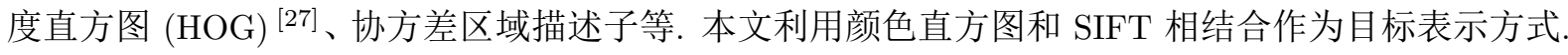
在没有光照改变、行人形变较大和遮挡的情况下, 视频中相邻两帧中的目标的颜色直方图的相似度较 高, 而且目标行人和其他非目标行人的颜色直方图相似度存在较大的差异, 这种情况下采用颜色直方 图来表示目标. 颜色直方图的主要优点在于计算速度快, 不会影响目标跟踪的实时性, 因此作为本文 跟踪框架的第 1 目标表示方式, SIFT 由于其精确性和实时性差, 作为跟踪框架的后备目标表示方式.

\section{3 颜色直方图匹配}

我们将对比的图像转换成 $256 \times 256$ 像素的相同大小的图片, 这样有利于计算出相像的特征, 然后 分别计算两张图像分别对应的 RGB 3 个通道的相似度, 最后取 3 个相似度的平均值作为两张图像的 最终相似度.

传统的颜色相似度计算方法虽然能够区分不同的目标, 但是区分度并不大 ${ }^{[28]}$, 从图 2 中可观察 到, 不同图像颜色直方图的主要区别在于直方图的波峰段, 为了利用这一特性, 本文提出一种新的相 似度计算方法, 利用均值 (图 2 中的黄线) 与直方图的交点取出直方图的波峰段, 然后计算每个波段的 相似度, 最后取均值. 在计算单通道的相似度时, 要先把像素值进行归一化处理, 然后采用式 (1) 计算 
表 1 两种方法的图像区分度对比

Table 1 The degree of differentiation between two methods

\begin{tabular}{ccccc}
\hline Method & Experiment (1) & Experiment (2) & Experiment (3) & Experiment (4) \\
\hline Tradition $^{[28]}(\%)$ & 41.8 & 33.3 & 35.4 & 28.5 \\
Proposed (\%) & 43.5 & 40.1 & 47.3 & 36.7 \\
\hline
\end{tabular}

相似度, 其中 $G, S$ 为单通道直方图, $N$ 为波峰段的个数, $m_{i}$ 和 $n_{i}$ 为每个波峰段的区间, $n_{i}-m_{i}$ 为每 个波段颜色空间样本点数.

本文中提出利用区分度来衡量相似度计算方法的性能. 其计算方法为式 (2), 其中 $S_{1}$ 表示目标与 相邻帧目标对象的相似度, $S_{2}$ 表示目标与同一帧其他非目标对象的相似度. 表 1 展示了传统方法和本 文提出的方法在 4 组实验中的对比, 很明显本文的方法提高了目标的区分度.

$$
\begin{aligned}
& \operatorname{Sim}(G, S)=\frac{1}{N} \sum_{i=1}^{N}\left(\frac{1}{n_{i}-m_{i}} \sum_{j=m_{i}}^{n_{i}}\left(1-\frac{\left|g_{j}-s_{j}\right|}{\operatorname{Max}\left(g_{j}, s_{j}\right)}\right)\right), \\
& D\left(S_{1}, S_{2}\right)=\frac{S_{1}-S_{2}}{S_{1}}, \\
& T= \begin{cases}0, & i=1, \\
\operatorname{Sub}\left(s_{1}, \ldots, s_{i}\right)+\left(\operatorname{Max}\left(s_{1}, \ldots, s_{i}\right)-\operatorname{Sub}\left(s_{1}, \ldots, s_{i}\right)\right) \times \lambda, & i>1 .\end{cases}
\end{aligned}
$$

实验表明, 相似度的大小受图像的像素大小和图像中的行人间的差异影响, 所以阈值不能是一个 固定的值, 对于不同的场景颜色直方图相似度的阈值都不一样. 在本文中, 利用式 (3) 来确定阈值, 如 果从第 1 帧开始就只有目标存在, 那么设定阈值为 0 , 如果存在两个以上的目标, 采用相似度的最大值 和次大值的平均值作为下一帧检测的阈值, 其中 $s_{i}$ 表示候选目标与目标模型的颜色直方图相似度, $i$ 表示在一帧图像中检测到的所有行人的个数.

\subsection{SIFT 匹配}

为了排除因为图像遮挡和背景混乱而产生的无匹配关系的关键点, SIFT 的作者 Lowe 提出了比 较最近邻距离与次近邻距离的 SIFT 匹配方式: 取一幅图像中的一个 SIFT 关键点, 并找出其与另一 幅图像中欧式距离最近的前两个关键点, 在这两个关键点中, 如果最近的距离除以次近距离得到的比 率 ratio 少于某个阈值 $T$, 则接受这一对匹配点. 因为对于错误匹配, 由于特征空间的高维性, 相似的 距离可能有大量其他的错误匹配, 从而它的 ratio 值比较高. 显然降低这个比例阈值 $T$, SIFT 匹配点 数目会减少, 但更加稳定, 反之亦然. Lowe 推荐 ratio 的阈值为 0.8 , 但作者对大量任意存在尺度、旋 转和亮度变化的两幅图片进行匹配, 图 3(a) 表示的是同一个人在视频中的不同位置的所有尺度不变 特征点. 结果表明 ratio 取值在 $0.4 \sim 0.6$ 之间最佳, 小于 0.4 的会减少正确的匹配点甚至很少有匹配 点, 大于 0.6 的则存在大量错误匹配点. 所以建议 ratio 的取值原则如下: 对于准确度要求高的匹配, ratio $=0.4$; 对于匹配点数目要求比较多的匹配, ratio $=0.6$. 一般情况下. 本文中 SIFT 特征匹配主要用 于寻找遮挡后出现的行人, 对准确度要求很高, 因此取 $r a t i o=0.4$. 鉴于 SIFT 的实时性差, 本文使用开 源工具包 VLFeat 提供的二进制文件来计算图像的 SIFT 特征.

在特征匹配的过程中, 我们认为目标模型背景的特征点是噪声点, 希望只匹配目标行人身体上的 特征点, 因为这些特征点才是目标独有的. 因此, 引入 FCN 图像语义分割来去除背景噪声. 如图 4 所

846 


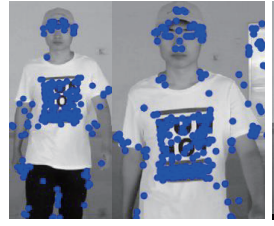

(a)

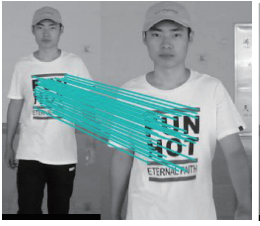

(b)

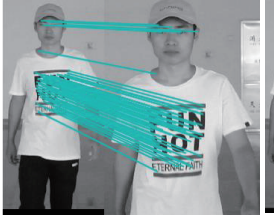

(c)

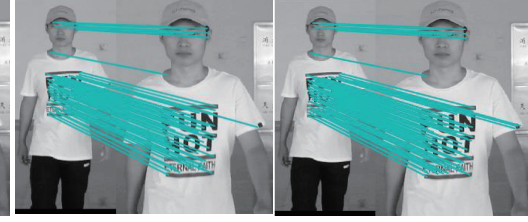

(d)

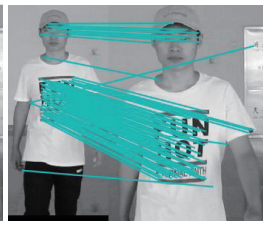

(f)

图 3 (网络版彩图) 在不同的阈值下同一个人在图像中的不同位置的特征匹配效果

Figure 3 (Color online) The effect of the feature matching for the same person in the different location in the video base on the different threshold. (a) SIFT feature points distribution of the same person; (b) ratio=0.3; (c) ratio=0.4; (d) ratio $=0.5 ;$ (e) ratio $=0.6$; (f) ratio $=0.8$

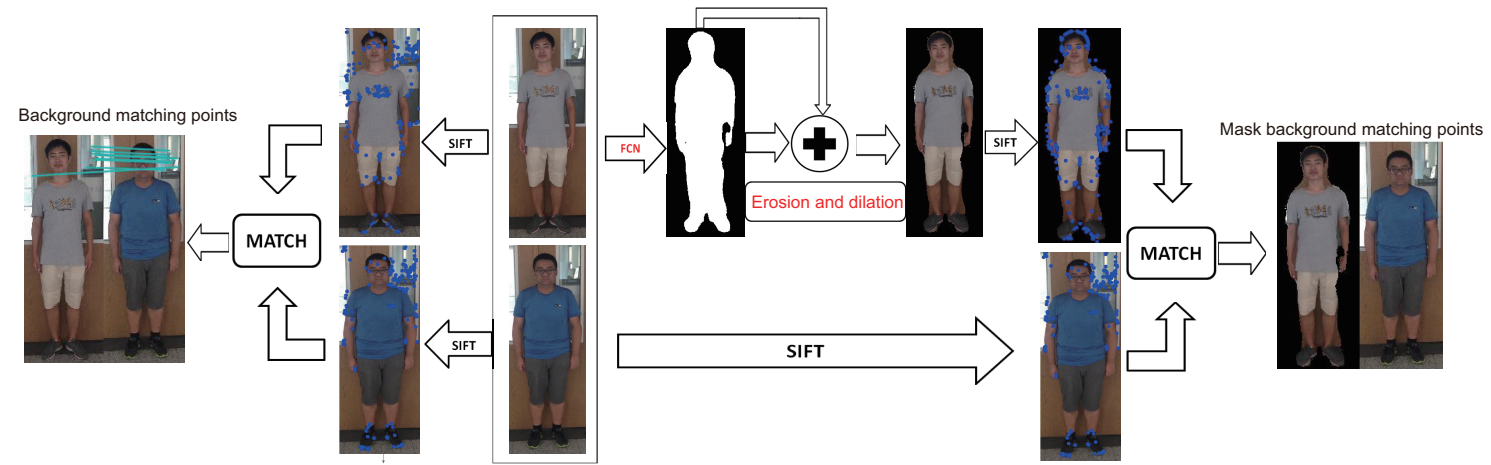

图 4 (网络版彩图) 去除行人图像背景中匹配点

Figure 4 (Color online) The decrease of the background matching points

示, 将目标模型输入 FCN 网络得到的结果和原图像结合达到祛除背景的目的, 从图中左右两方的对 比, 很容易发现这种方法能够去除背景对目标匹配的影响.

在本文中将 VGG-16 网络改为全卷积网络, 在检测行人的 CNN 结构中, 前 5 层是卷积层, 第 6,7 层 分别是一个长度为 4096 的一维向量, 第 8 层是长度为 2 的一维向量, 分别对应行人和背景的概率. 本 文将这 3 层转换为卷积层, 卷积核的大小 (通道数, 宽, 高) 分别为 $(4096,1,1),(4096,1,1),(2,1,1)$. 看 上去数字上并没有什么差别, 但是卷积跟全连接是不一样的概念和计算过程, 使用的是之前 CNN 已 经训练好的权重值和偏置, 不同之处在于权值和偏置是有自己的范围, 属于自己的一个卷积核. 因此 FCN 网络中所有的层都是卷积层, 故称为全卷积网络. 如图 5 所示, 本文将不同池化层的结果进行上 采样, 然后结合这些结果来优化输出.

接下来行人跟踪框架的整体流程. 框架的输入是一段视频或者是摄像机获取的图像. 首先使用预 先训练好的 Faster R-CNN 模型检测第 1 帧的所有行人, 从其中选择和初始目标颜色直方图相似度最 大的目标作为跟踪目标, 并把目标剪切下来保存为目标模型. 然后输入下一帧图像, 同样利用 Faster R-CNN 检测出图像中的所有行人 (也就是候选目标), 首先利用颜色直方图表示目标模型和所有候选 目标并计算候选目标和目标模型的相似度和阈值, 确认和目标模型相似度最大的候选目标为跟踪目标 并且更新为目标模型. 如果利用颜色直方图相似度对比的最大值小于设定的相似度阈值, 更改目标的 表示方法为 SIFT 特征. 将目标模型输入 FCN 网络去除目标模型的背景, 得到新的目标模型, 计算新 的目标模型和所有的候选目标的 SIFT 特征, 然后计算候选目标和目标模型特征匹配点的个数, 取其 中特征匹配数目最多的目标为最近似目标. 经过大量实验表明, 特征匹配数目超过 3 个以上一般被认 为是相似的图像. 如果最近似目标的特征匹配数目小于 3 , 认为目标已经在实验范围内消失, 目标模型 


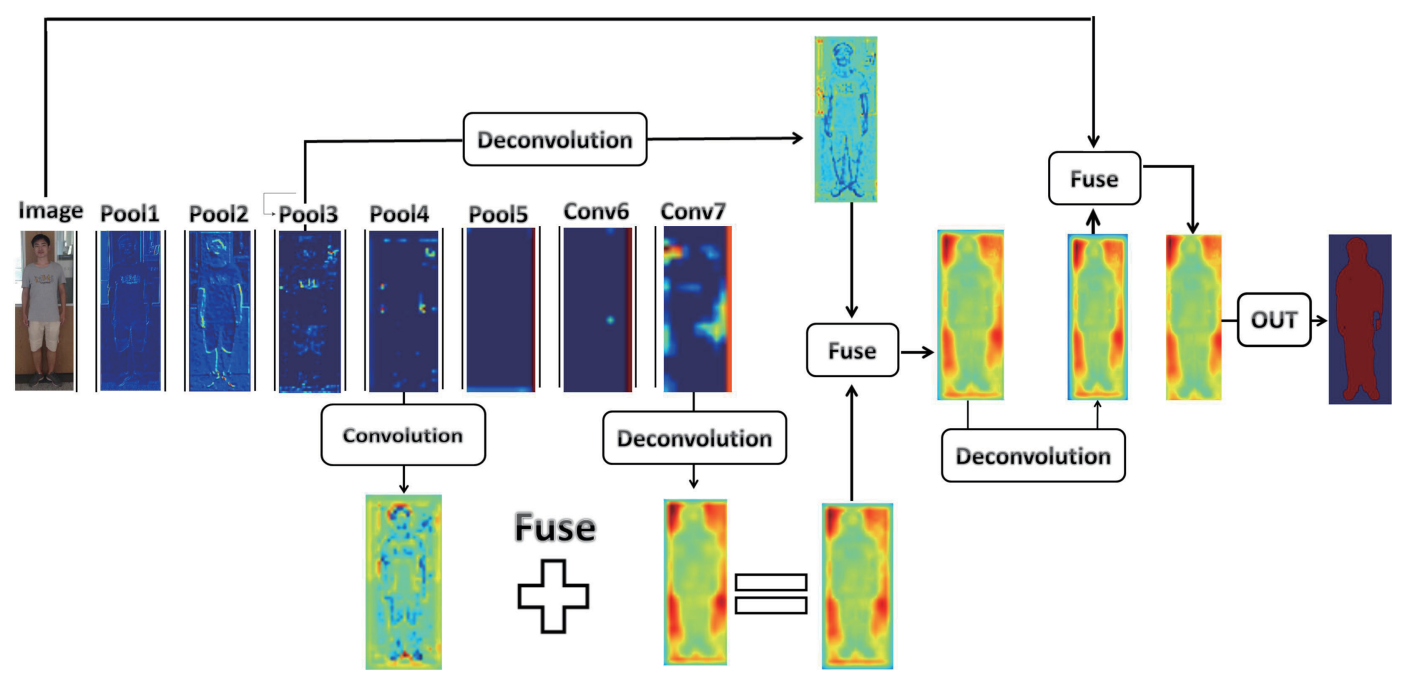

图 5 (网络版彩图) 全卷积网络的结构图

Figure 5 (Color online) The structure of the fully convolution network

不进行更新, 进入下一帧图像的目标跟踪, 重复上述过程. 框架的代码实现如算法 1 所示.

\section{4 实验结果及分析}

我们将对本文提出的跟踪框架和在第 2 节提到的优秀跟踪算法进行比较. 由于我们的跟踪目标 是行人而不是所有物体, 并且主要解决的是遮挡问题, 而被大家广泛使用的跟踪基准里面行人的视频 较少而且存在行人遮挡的场景也不多, 因此在本文的实验中, 有的视频是来自跟踪基准, 有的视频是 我们按照跟踪基准 [29] 上的视频制作规则制作的.

这些视频包含了具有代表性的遮挡情况, 包括无遮挡的简单场景、镜头抖动、全部遮挡、部分遮 挡、人群中的遮挡、行人遮挡. 具体的视频属性如表 2 所示, 其序列号分别与图 $6 \sim 9$ 中的图标顺序一 一对应. 本文的行人检测模型和图像语义分割模型是在深度学习框架 $\mathrm{Caffe}{ }^{[30]}$ 上实现的. 卷积网络在 单个具有 11 GB 内存的 NVIDIA GeForce GTX 1080Ti GPU 上进行了训练. 训练 Faster R-CNN 时使 用 ImageNet ${ }^{[31]}$ 预训练的 VGG-16 网络同时初始化 RPN 和 Fast R-CNN, 然后使用数据集调整模型 参数.

在图 6 中, 将本文的框架与其他 5 种跟踪算法的中心位置误差 (CLE) 进行比较. 可以看出, 本文 所提出的框架在 CLE 方面获得了稳定的性能, 这可以通过平滑的曲线看出. 相比之下, 在简单的场景 中 6 种跟踪方法的 CLE 基本相同, 而且基本都在 50 个像素长度以下, 在镜头抖动并且没有遮挡的情 况下 OCT-KCF 算法的表现要好很多, 但是一旦有遮挡存在的情况下, 其他 5 种算法从遮挡一开始就 几乎不能再重新定位目标了. 例如在 Pedestrian Occlusion 场景中, 从第 63 帧起丢失目标, 然而在第 73 帧的时候本文的框架能够重新定位目标, 而其他算法却不能这样做. 原因是本文提出的搜索机制和 目标模型跟其他算法不同. 首先, 本文采用的 Faster R-CNN 搜索行人, 使得搜索范围尽可能地包含跟 踪目标. 其次, 我们的模型结合 FCN 网络只提取出模型中的行人信息, 很好地忽略了背景的影响, 使 得目标即使是在长时间的遮挡后都能被找到, 这点在图 6 中的 Full Occlusion 中可以看出.

为充分分析算法性能, 图 7 展示了本文框架与其他算法对不同序列的目标跟踪偏移距离精度分 


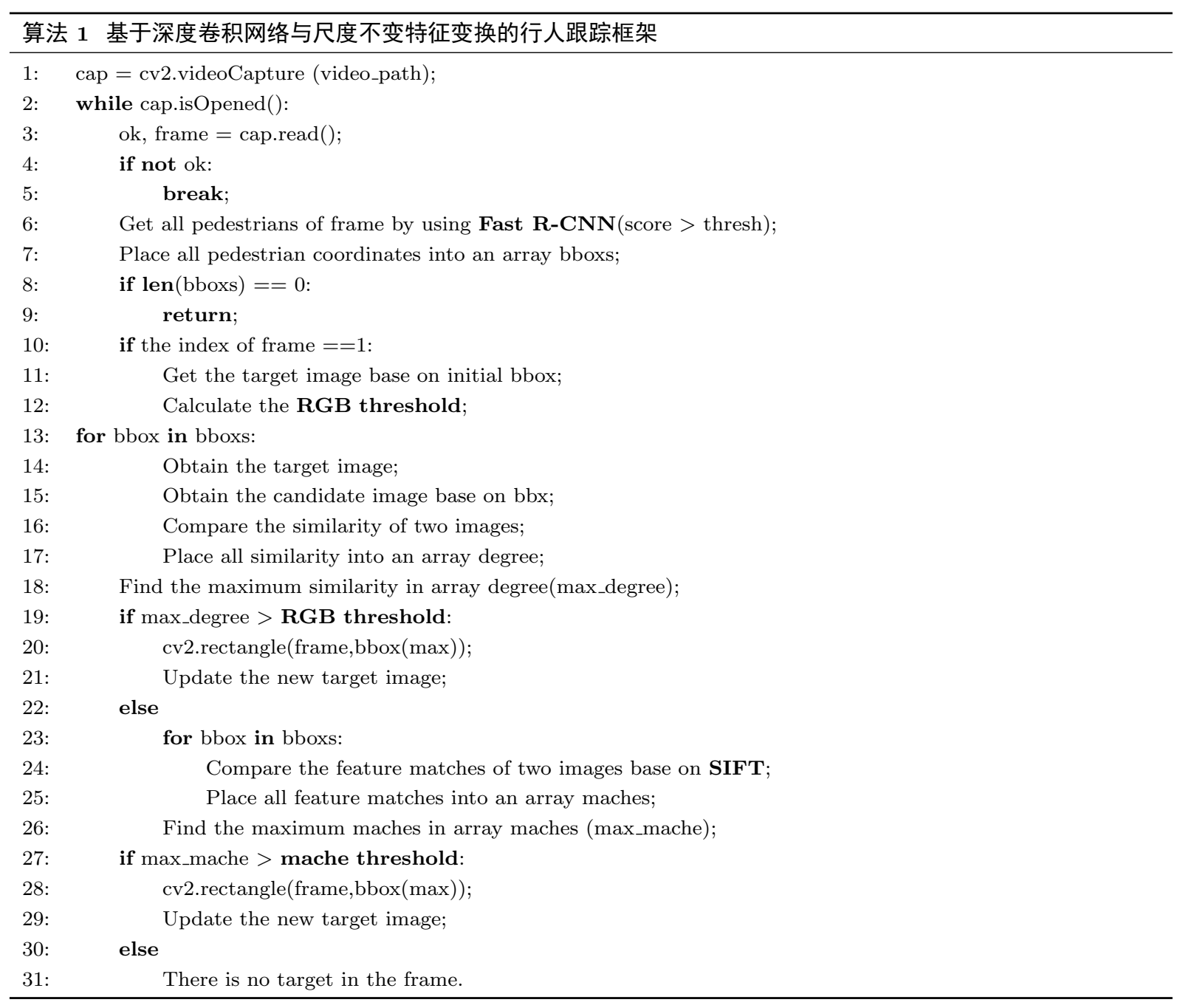

表 2 实验视频属性描述

Table 2 Video sequence attribute description

\begin{tabular}{cccccc}
\hline Video sequence & Occlusion & Illumination variations & Scale variations & Motion blur & Low resolution \\
\hline 1 & $\mathrm{~N}$ & $\mathrm{~N}$ & $\mathrm{~N}$ & $\mathrm{~N}$ & $\mathrm{~N}$ \\
2 & $\mathrm{~N}$ & $\mathrm{~N}$ & $\mathrm{Y}$ & $\mathrm{Y}$ & $\mathrm{Y}$ \\
3 & $\mathrm{Y}$ & $\mathrm{N}$ & $\mathrm{N}$ & $\mathrm{N}$ & $\mathrm{Y}$ \\
4 & $\mathrm{Y}$ & $\mathrm{N}$ & $\mathrm{N}$ & $\mathrm{N}$ & $\mathrm{Y}$ \\
5 & $\mathrm{Y}$ & $\mathrm{N}$ & $\mathrm{N}$ & $\mathrm{N}$ & $\mathrm{N}$ \\
6 & $\mathrm{Y}$ & $\mathrm{Y}$ & $\mathrm{N}$ & $\mathrm{Y}$ \\
7 & $\mathrm{Y}$ & $\mathrm{Y}$ & $\mathrm{N}$ & $\mathrm{N}$ \\
8 & $\mathrm{Y}$ & $\mathrm{Y}$ & $\mathrm{Y}$ & $\mathrm{N}$ & $\mathrm{N}$ \\
\hline
\end{tabular}

析. 横坐标表示跟踪中心位置偏移阈值, 单位为像素, 纵坐标表示跟踪精度 (这里只评估像素距离 50 以内的, 50 以外算作未跟踪到, 曲线表示检测到的目标偏移距离小于不同偏移阈值占总帧数的比例). 

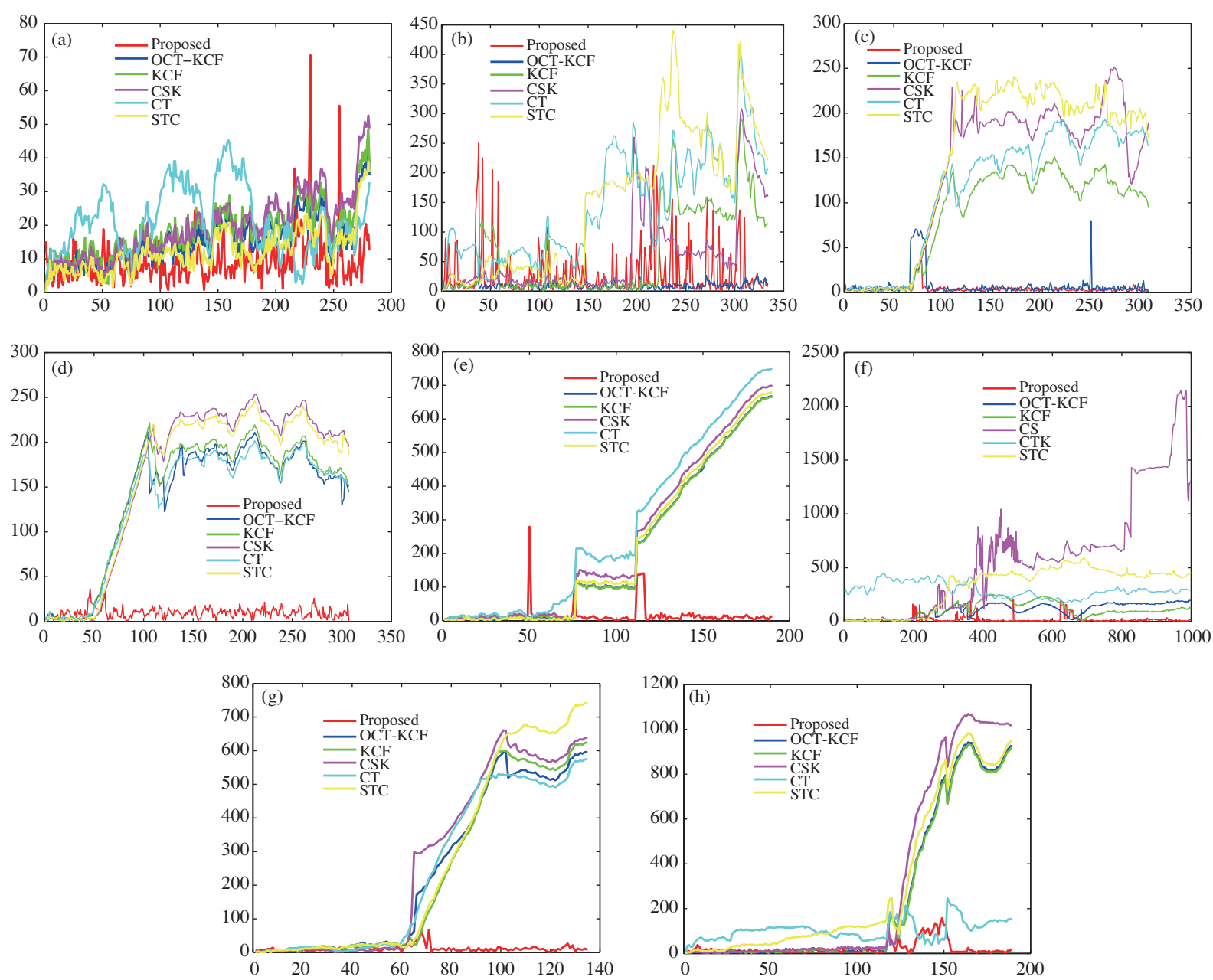

图 6 (网络版彩图) 本文框架和其他经典跟踪算法基于 CLE 的对比

Figure 6 (Color online) Comparison between proposed framework and other tracking algorithms based on CLE. Plots of CLE-out of (a) simple scene, (b) blurbody (no occlusion), (c) jogging 1 (occlusion), (d) jogging 2 (occlusion), (e) fully occlusion, (f) partial occlusion, (g) pedestrian occlusion, (h) multi-pedestrian occlusion

可见, 本文框架在没有遮挡的简单场景中和其他算法表现相近, 但是在具有遮挡的场景中表现明显 优异. 在镜头抖动的情况下精度低于 OCT-KCF 算法可能是由于来自跟踪 Benchmark 上的视频像素 太低.

本文采用文献 [32] 提出的成功率评估方法, 如图 8 所示, 横坐标表示目标矩形与理想标定矩形的 交叉比阈值 $S$ 的计算方法为式 (4) ( $R_{i}$ 表示跟踪矩形框, $R_{0}$ 表示理想矩形框), 纵坐标表示跟踪成功 率, 曲线表示所有检测目标的交叉大于不同阈值占总帧数的比率, 红线代表本文算法. 不同算法曲线 与纵坐标的交叉数值展示了目标在整个序列跟踪到的比率, 曲线的面积积分说明了算法跟踪成功的整 体性能. 从图 7 中可以明显看出, 与图 6 相对应本文提出的跟踪框架在有遮挡的情况下不仅跟踪的精 度高而且跟踪目标的成功率也明显优于其他跟踪算法.

$$
S=\frac{\left|R_{i} \cap R_{0}\right|}{\left|R_{i} \cup R_{0}\right|} .
$$



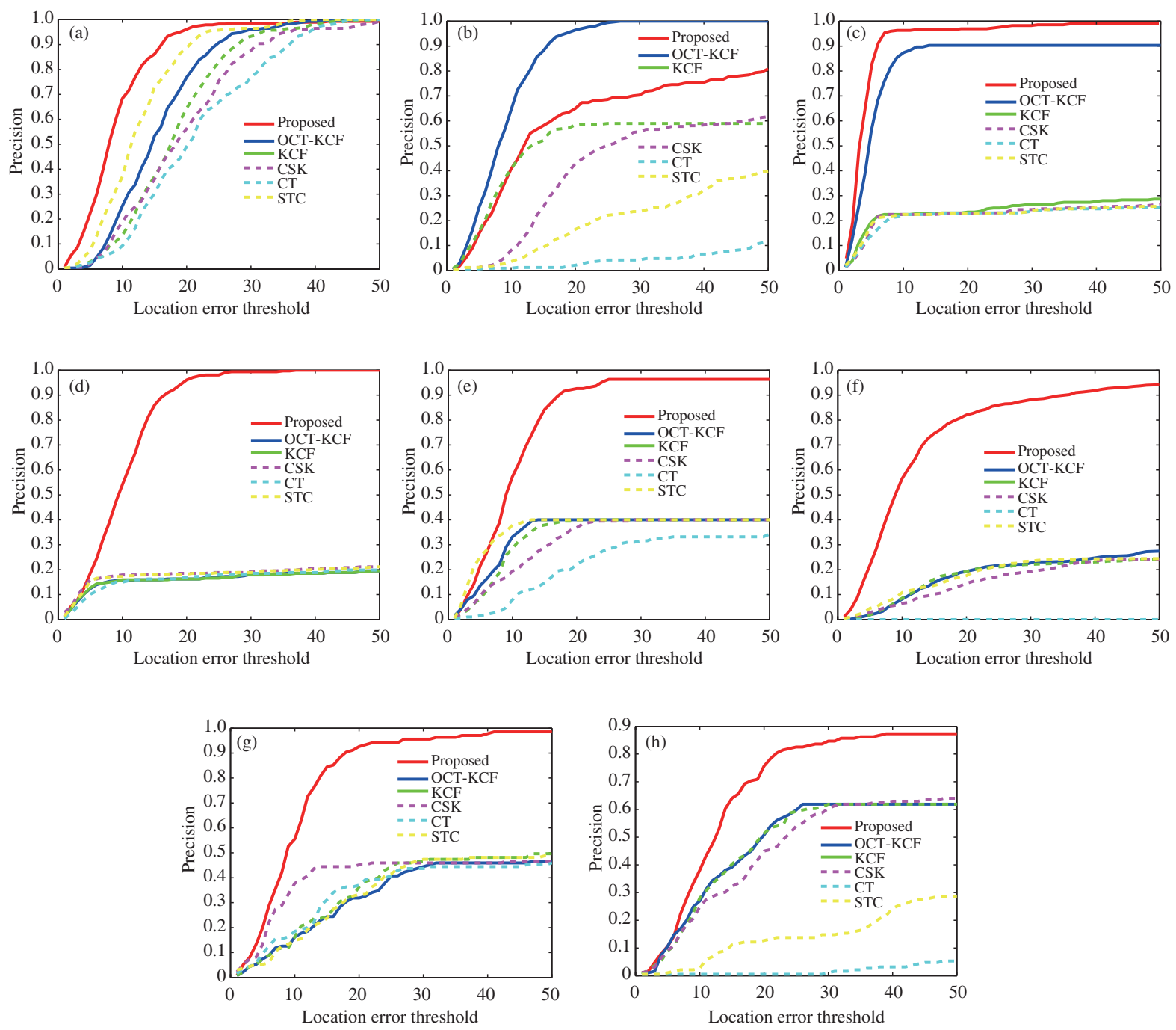

图 7 (网络版彩图) 8 种遮挡视频的准确率对比图

Figure 7 (Color online) Precision plots for the 8 attributes of the online tracking benchmark and our video with occlusion. Precision plots of OPE-out of (a) simple scene, (b) blurbody (no occlusion), (c) jogging 1 (occlusion), (d) jogging 2 (occlusion), (e) fully occlusion, (f) partial occlusion, (g) pedestrian occlusion, (h) multi-pedestrian occlusion

在图 9 中, 展示出了一些关键帧的跟踪结果. 第 1 行是一个简单的场景, 参与对比的所有跟踪算 法都取得不错的效果. 第 2 行是镜头抖动的情境, 其中 OCT-KCF 的跟踪效果较佳, 本文的跟踪算法 效果差的原因是跟踪 Benchmark 上的视频像素较低, 这也是本文的跟踪算法存在的问题, 有待以后优 化. 从第 3 行开始就是各种各样的遮挡情境. 相比之下, 所有其他比较的跟踪器在存在遮挡的情况下 基本都丢失目标, 本文的跟踪框架可以很好地在遮挡后重新定位目标.

\section{5 结论}

为了解决行人跟踪过程中的各种遮挡问题, 本文提出了预先训练一个行人检测器作为目标搜索机 

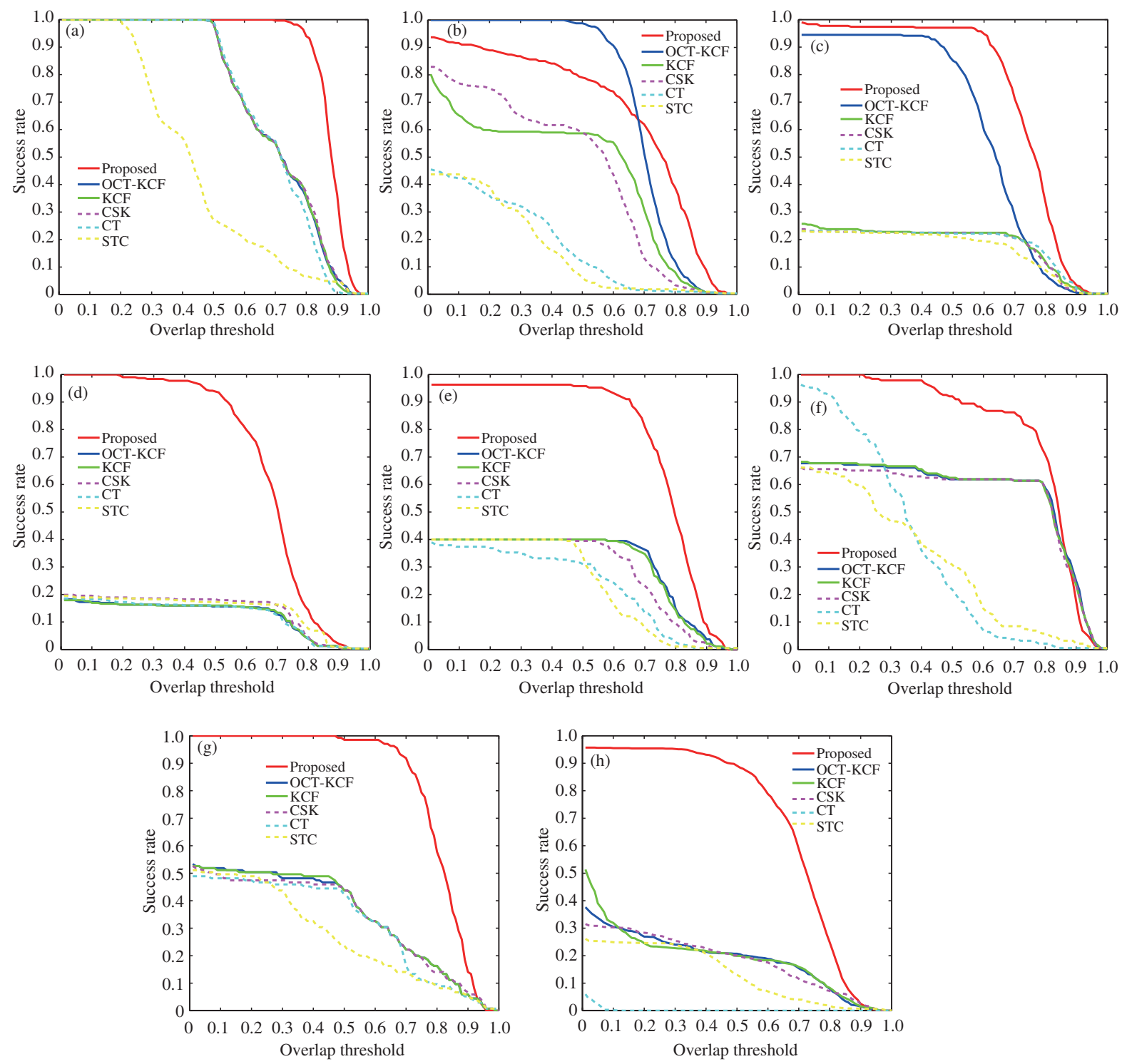

图 8 (网络版彩图) 8 种遮挡视频的成功率对比图

Figure 8 (Color online) Success plots for the 8 attributes of the online tracking benchmark and our video with occlusion. Success plots of OPE-out of (a) simple scene, (b) blurbody (no occlusion), (c) jogging 1 (occlusion), (d) jogging 2 (occlusion), (e) fully occlusion, (f) multi-pedestrian occlusion, (g) pedestrian occlusion, (h) partial occlusion

制, 然后用图像特征算法来区分目标和其他行人的跟踪框架. 本文的框架采用了当下对于目标检测性 能较好的 Faster R-CNN 作为目标搜索机制, 使得在行人跟踪过程中对行人的定位迅速准确. 本文框 架综合考虑了跟踪的实时性、准确性、对遮挡的鲁棒性等因素, 选择 RGB 直方图和 SIFT 相结合作为 目标表示方式. 切合实际地提出了新的 RGB 直方图相似度计算方法, 通过真实实验找到 SIFT 的最 佳匹配阈值并且成功地将 FCN 图像语义分割用于 SIFT 特征匹配的背景去噪. 对跟踪基准测试的广 泛实验和比较表明, 所提出的框架获得了比最先进的跟踪器更好的性能, 并且在处理高像素的图像时 实时性较高. 虽然获得了高性能, 但是在处理低像素的图像时, 由于 SIFT 特征较少, 会产生一定的漂 


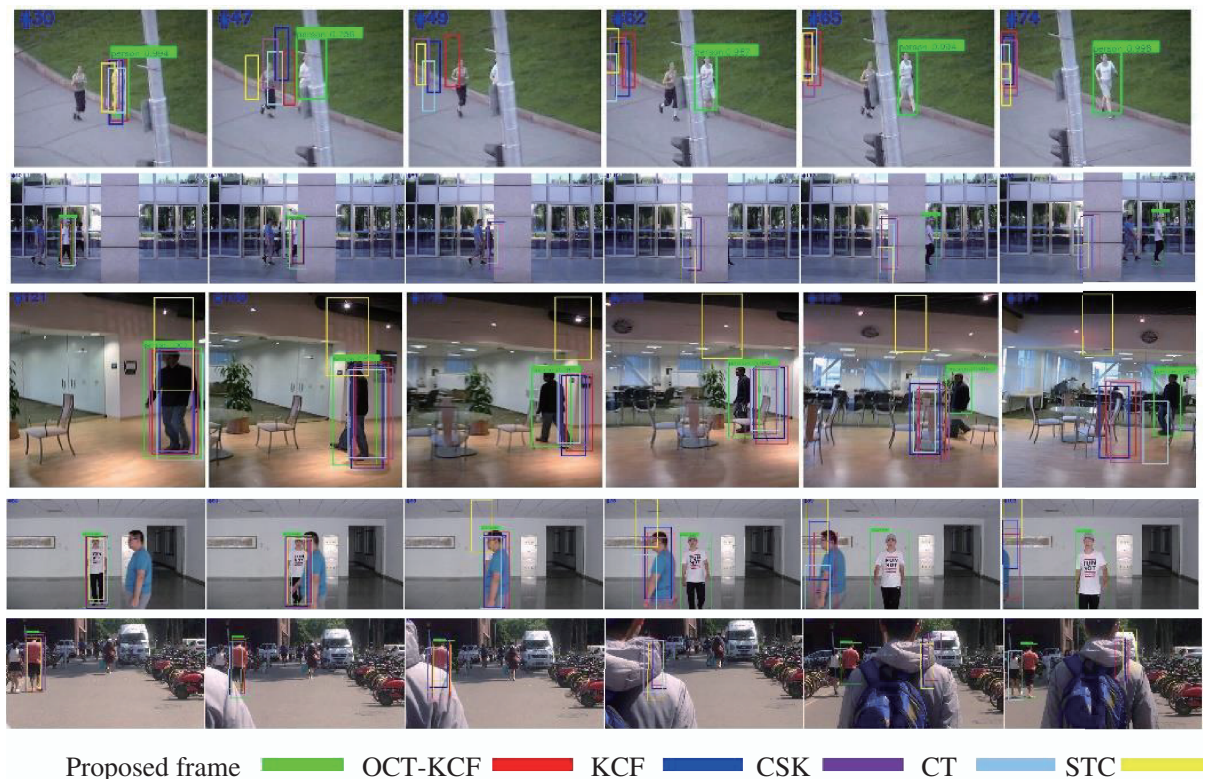

图 9 (网络版彩图) 一些关键帧的跟踪结果对比

Figure 9 (Color online) Illustration of some key frames

移现象. 因此, 未来的工作重点将放在寻找新的目标表示的目标匹配方法上, 以实现更高的跟踪性能. 此外, 还将尝试根据其他深度学习方法 (如文献 [33]) 来改进本文的框架, 以解决长期跟踪问题.

\section{参考文献}

1 Yilmaz A, Javed O, Shah M. Object tracking: a survey. ACM Comput Surv, 2006, 38: 81-93

2 Yao R, Shi Q F, Shen C H, et al. Part-based visual tracking with online latent structural learning. In: Proceedings of IEEE Conference on Computer Vision and Pattern Recognition, Portland, 2013. 2363-2370

3 Adam A, Rivlin E, Shimshoni I. Robust fragments-based tracking using the integral histogram. In: Proceedings of IEEE Computer Society Conference on Computer Vision and Pattern Recognition (CVPR'06), New York, 2006. 798-805

4 Ross D A, Lim J, Lin R S, et al. Incremental learning for robust visual tracking. Int J Comput Vision, 2008, 77: $125-141$

5 Zhuang B H, Lu H C, Xiao Z Y, et al. Visual tracking via discriminative sparse similarity map. IEEE Trans Image Process, 2014, 23: 1872-1881

6 Comaniciu D, Meer P. Mean shift: a robust approach toward feature space analysis. IEEE Trans Pattern Anal Mach Intel, 2002, 24: 603-619

7 Comaniciu D, Ramesh V, Meer P. Real-time tracking of non-rigid objects using mean shift. In: Proceedings of IEEE Conference on Computer Vision and Pattern Recognition, Hilton Head Island, 2002

8 Jin X B, Du J J, Bao J. Data-driven tracking based on Kalman filter. Appl Mech Mater, 2012, 226: 2476-2479

9 Ren S Q, He K M, Girshick R, et al. Faster R-CNN: towards real-time object detection with region proposal networks. IEEE Trans Pattern Anal Mach Intel, 2017, 39: 1137-1149

10 Lowe D G. Distinctive image features from scale-invariant keypoints. Int J Comput Vision, 2004, 60: 91-110

11 Bouman C A. Similarity-based retrieval of images using color histograms. In: Proceedings of Storage and Retrieval for Image and Video Databases, San Jose, 1998

12 Shelhamer E, Long J, Darrell T. Fully convolutional networks for semantic segmentation. IEEE Trans Pattern Anal Mach Intel, 2017, 39: 640-651

13 Zhang K H, Zhang L, Yang M H. Real-time compressive tracking. In: Proceedings of European Conference on 
Computer Vision, Florence, 2012

14 Zhang K H, Zhang L, Yang M H, et al. Fast tracking via spatio-temporal context learning. Comput Sci, 2013. ArXiv:1311.1939

15 Henriques F, Caseiro R, Martins P, et al. Exploiting the circulant structure of tracking-by-detection with kernels. In: Proceedings of European Conference on Computer Vision, Florence, 2012

16 Henriques J F, Caseiro R, Martins P, et al. High-speed tracking with kernelized correlation filters. IEEE Trans Pattern Anal Mach Intel, 2015, 37: 583-596

17 Zhang B C, Li Z G, Cao X B, et al. Output constraint transfer for kernelized correlation filter in tracking. IEEE Trans Syst Man Cybern Syst, 2017, 47: 693-703

18 Girshick R. Fast R-CNN. In: Prococeedings of IEEE International Conference Computer Vision, Santiago, 2015. 1440-1448

19 Ma J Q, Shao W Y, Ye H, et al. Arbitrary-oriented scene text detection via rotation proposals. IEEE Trans Multimedia, 2018. doi: 10.1109/TMM.2018.2818020

20 He K M, Zhang X Y, Ren S Q, et al. Spatial pyramid pooling in deep convolutional networks for visual recognition. IEEE Trans Pattern Anal Mach Intel, 2015, 37: 1904-1916

21 Li X, Hu W M, Shen C H, et al. A survey of appearance models in visual object tracking. ACM Trans Intel Syst Technol, 2013, 4: 1-48

22 Lucas B D, Kanade T. An iterative image registration technique with an application to stereo vision. In: Proceedings of the 7th International Joint Conference on Artificial Intelligence, Vancouver, 1981

23 Baker S, Matthews I. Lucas-Kanade 20 years on: a unifying framework. Int J Comput Vision, 2004, 56: 221-255

24 Hager G D, Belhumeur P N. Efficient region tracking with parametric models of geometry and illumination. IEEE Trans Pattern Anal Mach Intel, 1998, 20: 1025-1039

25 Matthews L, Ishikawa T, Baker S. The template update problem. IEEE Trans Pattern Anal Mach Intel, 2004, 26: $810-815$

26 Alt N, Hinterstoisser S, Navab N. Rapid selection of reliable templates for visual tracking. In: Proceedings of IEEE Computer Society Conference on Computer Vision and Pattern Recognition, San Francisco, 2010

27 Dalal N, Triggs B. Histograms of oriented gradients for human detection. In: Proceedings of IEEE Conference on Computer Vision and Pattern Recognition, San Diego, 2005. 886-893

28 Simonyan K, Zisserman A. Very deep convolutional networks for large-scale image recognition. In: Proceedings of International Conference on Learning Representations, San Diego, 2015

29 Wu Y, Lim J, Yang M H. Online object tracking: a benchmark. In: Proceedings of IEEE International Conference Computer Vision and Pattern Recognition, Portland, 2013. 2411-2418

30 Jia Y Q, Shelhamer E, Donahue J, et al. Caffe: convolutional architecture for fast feature embedding. In: Proceedings of the 22nd ACM International Conference on Multimedia, Orlando, 2014

31 Deng J, Dong W, Socher R, et al. Imagenet: a large-scale hierarchical image database. In: Proceedings of IEEE International Conference on Computer Vision and Pattern Recognition, Miami, 2009. 248-255

32 Wu Y, Lim J, Yang M H. Online object tracking: a benchmark. In: Proceedings of IEEE International Conference on Computer Vision and Pattern Recognition, Portland, 2013. 2411-2418

33 Nam H, Han B. Learning multi-domain convolutional neural networks for visual tracking. In: Proceedings of IEEE Conference on Computer Vision and Pattern Recognition, Las Vegas, 2016. 4293-4302 


\title{
Pedestrian tracking framework based on deep convolution net- work and SIFT
}

\author{
Kai CHEN, Xiao SONG* \& Jing LIU \\ School of Automation Science and Electrical Engineering, Beihang University, Beijing 100191, China \\ * Corresponding author. E-mail: songxiao@buaa.edu.cn
}

\begin{abstract}
Numerous object-tracking and multiple people-tracking algorithms have been put forward in the field of computer vision, but these algorithms did not address the issue of when a pedestrian is partially or fully occluded by another object or person. To achieve efficient pedestrian tracking in various occlusion conditions, this research presents a pedestrian-tracking framework based on deep learning. First, a pedestrian detector was trained as a pedestrian-tracking search mechanism based on the object detection algorithm Faster R-CNN, which narrows the search range and improves accuracy efficiently compared with traditional gradient-down algorithms. Second, color histogram and scale-invariant features transform (SIFT) were combined as the target model expression. In the process of target matching, a full convolution network (FCN) was trained for pedestrians to extract the pedestrian information in the target model base on an FCN image semantic segmentation algorithm for removing the background noise. Finally, extensive experiments on OTB demonstrate that the proposed method achieves better performance than other state-of-the-art trackers for precision and success rate.
\end{abstract}

Keywords Faster R-CNN, SIFT, FCN, image semantic segmentation, color histogram

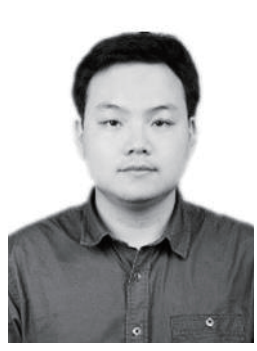

Kai CHEN was born in Shanxi, China, in 1991. He is a Ph.D. student of Beihang University, Beijing, China. His research interests include object tracking and machine and deep learning.

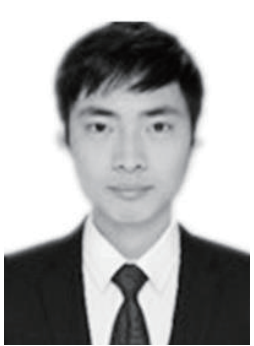

Jing LIU was born in Shandong, China, in 1994. He is a graduate student at Beihang University, Beijing, China. His research interests include pedestrian tracking and deep learning.

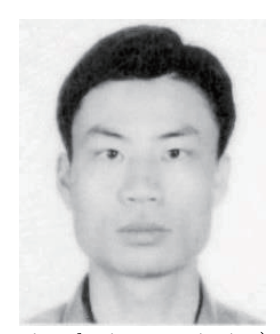

Xiao SONG received a Ph.D. degree in electrical engineering from Beihang University, Beijing, China, in 2006. He is currently an associate professor at the Automation School, Beihang University, China. He was a visiting researcher at the School of Computing, National University of Singapore between 2013 and 2014. He is a member of the ASIASIM (the federation of Asia simulation societies) council meeting. His research interests include pedestrian modeling and simulation and cloud computing. 